\title{
CALCULATION OF PERMEABILITY PARAMETER OF PERFORATED WALL
}

\author{
Kyung-Duck Suh, ${ }^{1}$ Yeul Woo Kim ${ }^{1}$ and Chang-Hwan $\mathrm{Ji}^{1}$
}

The friction coefficient in the permeability parameter of a perforated wall has been estimated on the basis of a best fit between measured and predicted values of such hydrodynamic coefficients as reflection and transmission coefficients. In the present study, an empirical formula for the friction coefficient is proposed in terms of known variables, i.e., the porosity and thickness of the perforated wall and the water depth. This enables direct estimation of the friction coefficient without invoking a best fit procedure. To validate the proposed formula, the reflection and transmission coefficients calculated by using the formula are compared with the experimental data for various types of structures including a perforated wall. It is also shown that the proposed formula can be used for irregular waves as well.

Keywords: perforated wall; permeability parameter; wave reflection; wave transmission

\section{INTRODUCTION}

During the past several decades, various types of breakwaters involving perforated walls have been introduced to resolve problems associated with gravity-type breakwaters, e.g., see Fig. 1 for a pile breakwater (or a perforated wall with vertical slits). Accordingly, mathematical models have also been developed to calculate the hydrodynamic characteristics such as wave reflection, transmission, and force at the perforated wall. In most of the models, the fluid region is divided into the front and back ( $\Omega_{1}$ and $\Omega_{2}$ in Fig. 1) of the perforated wall, solutions are assumed in each region, and they are obtained using the matching condition at the perforated wall. In Fig. $1, h=$ water depth, $b=$ thickness of the wall, and $H_{i}=$ incident wave height. A Cartesian coordinate system $(x, z)$ is defined with the positive $x$ directing downwave from the crest line of the breakwater and the vertical coordinate $z$ being measured vertically upwards from the still water line. The distance between the centers of two neighboring piles is denoted as $2 A$ and the width of an opening is $2 a$ so that the porosity of the wall is defined as $r=a / A$.

The matching condition can be expressed as

$$
\frac{\partial \phi_{1}}{\partial x}=\frac{\partial \phi_{2}}{\partial x}=i G\left(\phi_{1}-\phi_{2}\right) \quad \text { at } \quad x=0
$$

in which $\phi(x, z)$ is the spatial variation of the velocity potential. This matching condition describes that the horizontal velocities in the two regions must be same at the wall and that the horizontal velocity at the slit is proportional to the difference of velocity potentials, or the pressure difference, across the wall. The proportional constant $G$, often called the permeability parameter, is in general complex.

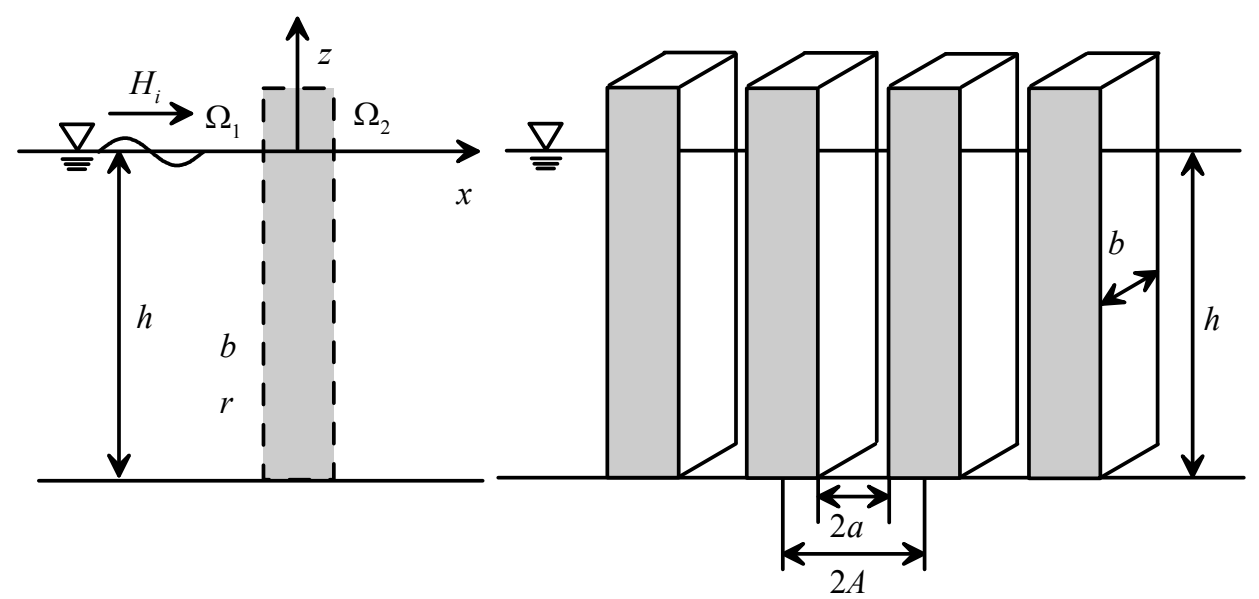

Figure 1. Definition sketch of pile breakwater; left : side view, right : bird's eyes view.

\footnotetext{
${ }^{1}$ Department of Civil and Environmental Engineering, Seoul National University, 599 Gwanak-no, Gwanak-gu, Seoul 151-744, Republic of Korea. kdsuh@snu.ac.kr
} 
The permeability parameter is usually calculated by the method of Kim (1998) or Yu (1995). Each method is based on Mei et al.'s (1974) and Sollitt and Cross's (1972) formulations, respectively. The former expresses the resistance and inertial effect in terms of head loss coefficient and effective orifice length, respectively, while the latter in terms of friction coefficient and added mass coefficient, respectively. In the Kim's (1998) method, the permeability parameter is expressed in terms of known variables. This is the advantage of the method. However, Suh et al. (2006) found that this method gives a wrong result for shallow water waves. The Yu's (1995) method gives correct results in all water depths. However, suitable values of the friction and added mass coefficients are in general not known a priori. Usually the friction coefficient has been estimated on the basis of a best fit between measured and predicted values of various hydrodynamic coefficients with a fixed added mass coefficient. In this study, we only deal with the Yu's (1995) method.

The Yu's (1995) formula is expressed as

$$
G=\frac{r}{b(f-i s)}
$$

in which $i=\sqrt{-1}, f=$ friction coefficient, and $s=1-C_{m}(1-r) / r$ is the inertia coefficient, where $C_{m}$ is the added mass coefficient. Many researchers (e.g. Sollitt and Cross 1972; Losada et al. 1993; Yu 1995; Isaacson et al. 1998; Zhu and Chwang 2001; Hossain et al. 2001) proposed to use the value of $C_{m}=0$, or $s=1$ through comparisons between predicted and experimental values of reflection and transmission coefficients of perforated structures. Recently Li et al. (2006) proposed a formula to calculate the friction coefficient as a function of $b / h$. In the present paper, we show that the porosity of the wall is also an important variable and propose a formula for the friction coefficient as a function of $r b / h$.

\section{LABORATORY EXPERIMENTS}

To obtain an empirical formula for $f$ as a function of $r b / h$, hydraulic experiments were carried out in the wave flumes in Seoul National University (SNU hereinafter) and Korea Ocean Research and Development Institute (KORDI hereinafter). Fig. 2 shows a perforated wall and wave gauges installed in the wave flume in KORDI.

The wave flume at the Department of Civil and Environmental Engineering of SNU was $30 \mathrm{~m}$ long, $0.6 \mathrm{~m}$ wide, and $1.0 \mathrm{~m}$ deep. It was equipped with a piston-type wave maker at one end, and a wave absorbing beach at the other end. Square piles of side lengths of 2 or $3 \mathrm{~cm}$ were used to make two different perforated walls. A constant porosity of $r=0.1$ was used for each perforated wall. The perforated wall was placed at a distance of $18.5 \mathrm{~m}$ from the wave maker. Nine different water depths were used from 30 to $70 \mathrm{~cm}$ with an increment of $5 \mathrm{~cm}$. In each water depth, six different wave periods $(T=1.0,1.2,1.4,1.6,1.8,2.0 \mathrm{~s})$ were used with specified wave heights corresponding to a constant wave steepness, $H / L=0.03$.

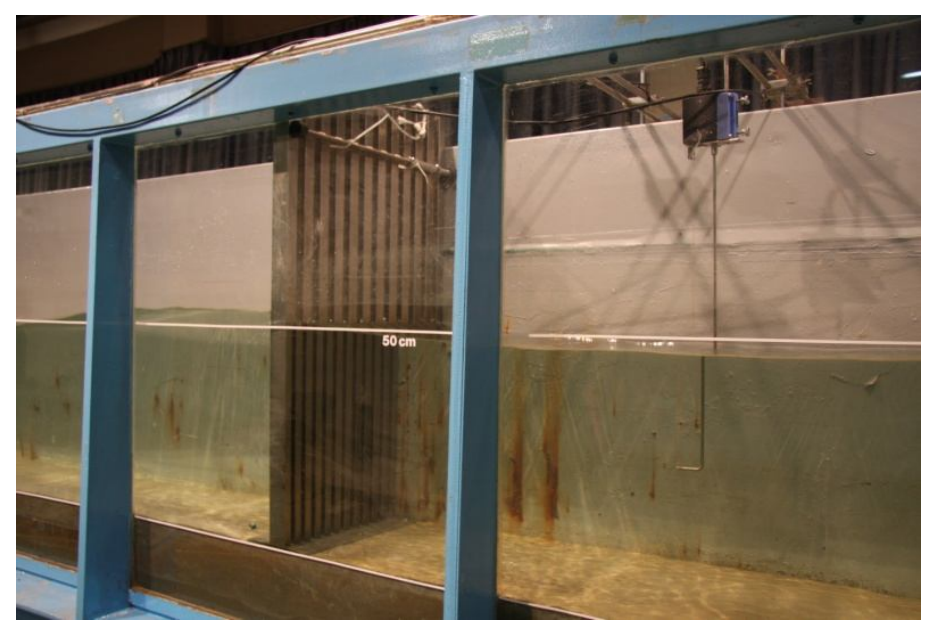

Figure 2. Perforated wall and wave gauges installed in wave flume in KORDI. 
To measure the incident and reflected waves, three wave gages were installed at a distance of about $4 \mathrm{~m}$ in front of the perforated wall. The free surface displacements measured by these wave gages were used to separate the incident and reflected waves using the method of Suh et al. (2001). For the purpose of cross-check, the incident waves were also measured at a distance of $4 \mathrm{~m}$ from the wave maker, where several well-established incident waves could be measured without being contaminated by the waves reflected from the perforated wall. The transmitted waves were measured using one wave gage located at a distance of $3.5 \mathrm{~m}$ behind the perforated wall assuming that the wave reflection from the downwave beach is negligible. Previous observation indicated reflection coefficients from the beach smaller than 0.1 for the wave periods used in these tests. Wave measurements were made for $75 \mathrm{~s}$ at a sampling rate of $20 \mathrm{~Hz}$ immediately after the initiation of wave generation at each of the wave gages.

In the SNU experiment, a single porosity was used with only square piles. More tests were conducted in KORDI for various porosities with both square and rectangular piles. The wave flume at the Coastal Engineering \& Ocean Energy Research Department of KORDI was $53 \mathrm{~m}$ long, $1.0 \mathrm{~m}$ wide, and $1.25 \mathrm{~m}$ deep. It was also equipped with a piston-type wave maker at one end, and a wave absorbing beach at the other end. The test section was divided into two channels by a vertical wall along the flume, each has a width of $0.6 \mathrm{~m}$ and $0.4 \mathrm{~m}$, respectively. The perforated wall was installed in the wider channel at a distance of $40 \mathrm{~m}$ from the wave maker, and the other channel was left empty. Rectangular or square piles were used to make the perforated walls. Two different widths of the pile were used: $2(A-a)=2 \mathrm{~cm}$ and $3 \mathrm{~cm}$. For the $2 \mathrm{~cm}$ wide pile, two different thicknesses were used: $b=2 \mathrm{~cm}$ and $4 \mathrm{~cm}$. For the $3 \mathrm{~cm}$ wide pile, three different thicknesses were used: $b=1 \mathrm{~cm}, 3 \mathrm{~cm}$, and $5 \mathrm{~cm}$. For each of these piles, the pile spacing $2 a$ was varied to make the porosity to be $0.2,0.3$, or 0.4 . In total, fifteen different perforated walls were used, i.e. $(2+3) \times 3=15$. Three different water depths were used: $h=40 \mathrm{~cm}, 50 \mathrm{~cm}$, and $60 \mathrm{~cm}$. In each water depth, six different wave periods $(T=1.0,1.2,1.4$, $1.6,1.8,2.0 \mathrm{~s}$ ) were used with specified wave heights corresponding to a constant wave steepness, $H / L=0.03$.

To measure the incident and reflected waves, three wave gages were installed at a distance of about $5 \mathrm{~m}$ in front of the perforated wall in the wider channel. The free surface displacements measured by these wave gages were used to separate the incident and reflected waves using the method of Suh et al. (2001). The incident waves were also measured using one wave gauge in the narrower channel which was left empty. The incident wave height measured in the narrower channel was used in the calculation of wave reflection and transmission coefficients. The transmitted waves were measured using one wave gage located at a distance of $3 \mathrm{~m}$ behind the perforated wall assuming that the wave reflection from the downwave beach is negligible. Wave measurements were made for $100 \mathrm{~s}$ at a sampling rate of $20 \mathrm{~Hz}$ immediately after the initiation of wave generation at each of the wave gages.

\section{EMPIRICAL FORMULA}

We seek an empirical formula for the friction coefficient $f$ expressed as a function of $r b / h$. As described in the previous section, tests were performed for 63 cases in total: 18 cases in SNU experiment ( 9 water depths for 2 perforated walls) and 45 cases in KORDI experiment ( 3 water depths for 15 perforated walls). For each test case, wave reflection and transmission coefficients were measured for six different waves. The corresponding reflection and transmission coefficients were calculated using the mathematical model using the eigenfunction expansion method, the details of which can be found in Isaacson et al. (1998) and Suh et al. (2006) among others. To find the friction coefficient that gives the best agreement between mathematical model and experimental results, we calculated the reflection and transmission coefficients by changing the friction coefficient from 0.1 to 10.0 at an increment of 0.1 . The friction coefficient that yields the largest index of agreement was then selected. The index of agreement is a statistical parameter proposed by Willmott (1981) as a measure of the degree to which a model's predictions are error-free. It varies between 0 and 1.0, where 1.0 indicates perfect agreement between observation and prediction, and 0 connotes complete disagreement. The indices of agreement for the selected friction coefficients varied between 0.345 and 0.999 with the average of 0.878. Fig. 3 shows an example of comparison between measured and predicted reflection and transmission coefficients as functions of $k h$. In this case, $f=0.6$ gives the best agreement.

It may be worthwhile to compare the present result with that of other researchers and propose an empirical formula by combining these results. We calculated the friction coefficient $f$ as a function of $r b / h$ using the aforementioned method for the data of Hagiwara (1984), Kriebel (1992), Kakuno and 
Liu (1993), Isaacson et al. (1998), Cho and Kim (2002), Li et al. (2006), and Huang (2007a,b). Fig. 4 shows the relationship between $f$ and $r b / h$ obtained using both present and other researchers' experimental results. Other researchers' results do not show big difference from the present result except Li et al.'s (2006) result, which gives larger friction coefficients than others. The reason is not known. The best-fit curve excluding the Li et al.'s data is given by

$$
f=0.0584\left(\frac{r b}{h}\right)^{-0.7}
$$

with the correlation coefficient of 0.795 .

At the end of the introduction, we have mentioned that there was a previous study in which the friction coefficient was expressed as a function of $b / h$. Fig. 5 shows the scatter plot of $f$ versus $b / h$. Compared with Fig. 4, more scattering of the data is observed. The same data were used in the two figures. Therefore, we could say that the friction coefficient is better expressed as a function of $r b / h$ rather than $b / h$.
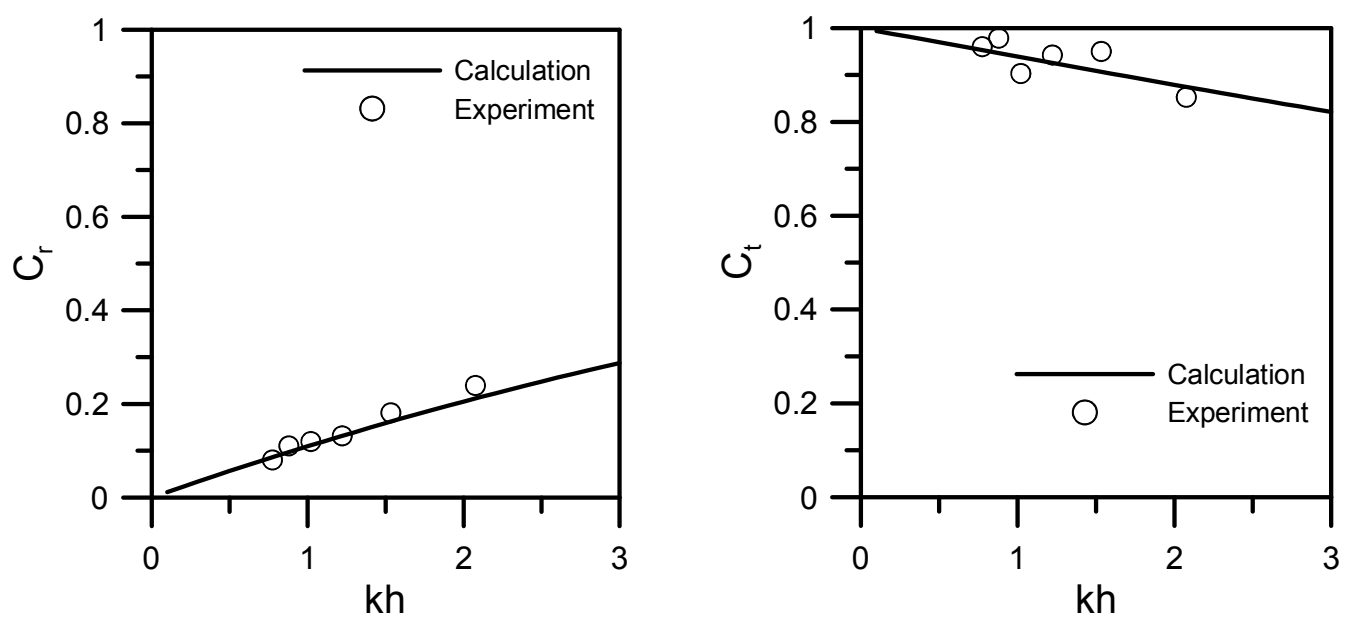

Figure 3. Example of comparison of predicted wave reflection and transmission coefficients with experimental results as functions of $k h ; 2(A-a)=2 \mathrm{~cm}, b=4 \mathrm{~cm}, r=0.4, h=50 \mathrm{~cm}, f=0.6$, and index of agreement $=0.999$.

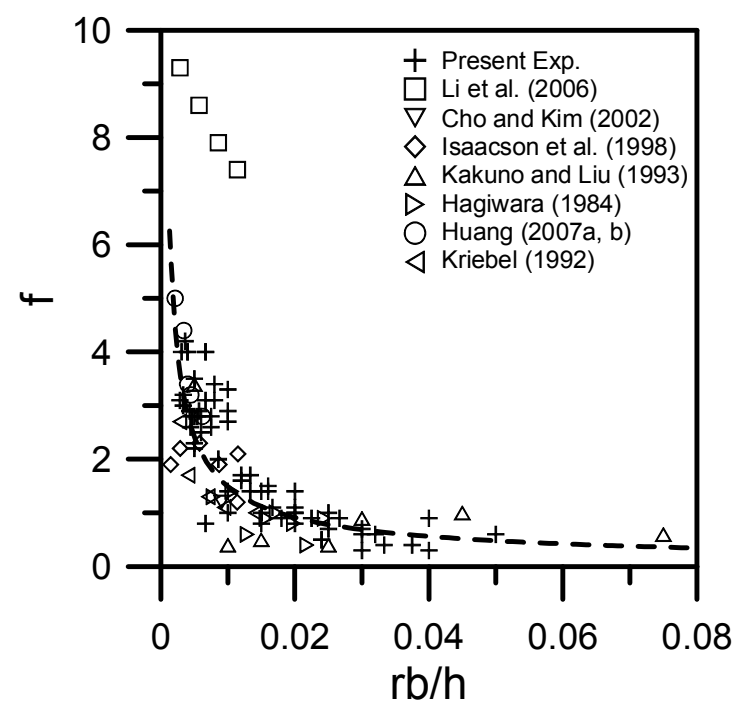

Figure 4. Relationship between $r b / h$ and $f$. 


\section{VALIDATION}

In order to validate the proposed empirical formula, the mathematical model results were compared with the experimental results of pile breakwaters, which consist of an array of vertical piles as shown in Fig. 1. Fig. 6 shows a comparison between measurement and prediction of the wave reflection or transmission coefficients of the present and other researchers' experiments. Reasonable agreement is shown between measurement and prediction, though the accuracy of the mathematical model tends to be lower near the middle range of the coefficients, i.e., $0.3 \leq C_{r}, C_{t} \leq 0.7$.

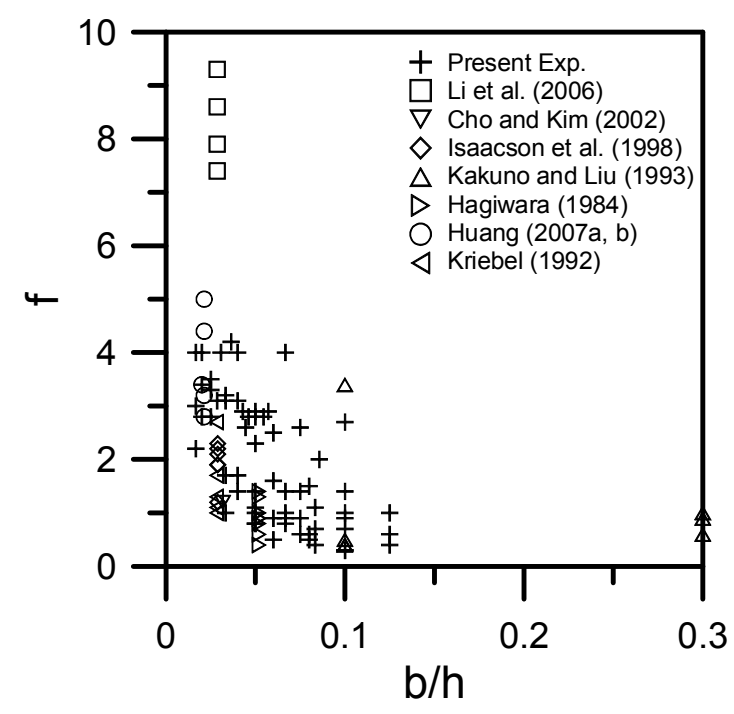

Figure 5. Scatter plot of $f$ versus $b / h$.

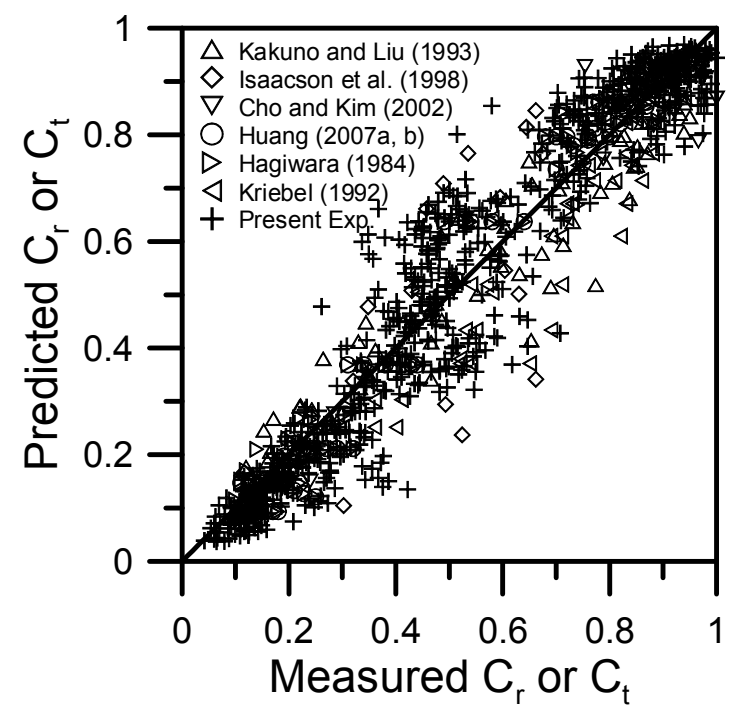

Figure 6. Comparison between predicted and measured reflection or transmission coefficients of pile breakwaters. 
For further validation of the empirical formula, it was applied to a curtain-wall-pile breakwater as shown in Fig. 7, the upper part of which is an impermeable curtain wall and the lower part is a perforated wall consisting of an array of vertical piles. The experimental data of Suh et al. (2006) was used. Their experiment was conducted in a large wave flume at the O. H. Hinsdale Wave Research Laboratory of Oregon State University, which was $104 \mathrm{~m}$ long, $3.7 \mathrm{~m}$ wide, and $4.6 \mathrm{~m}$ deep. The water depth was $2.4 \mathrm{~m}$. Square piles were used for the perforated wall, with $a=7.15 \mathrm{~cm}, A=14.3 \mathrm{~cm}$, and $b=14.3 \mathrm{~cm}$, so that the porosity of the lower perforated wall was $r=0.5$. Therefore, the friction factor is calculated as $f=0.7$. Three different drafts of the upper impermeable wall were used: $d=48$, 96 , and $144 \mathrm{~cm}$.

Fig. 8 compares the measured and predicted reflection and transmission coefficients as functions of $k h$. The readers are referred to the paper of Suh et al. (2006) for the construction of the mathematical model, which is the same as that used for a pile breakwater except for the additional matching condition at the upper curtain wall (i.e., $\partial \phi_{1} / \partial x=\partial \phi_{2} / \partial x=0$ for $-d \leq z \leq 0, x=0$ ). In general, the mathematical model adequately reproduces most of the important features of the experimental results, even though it slightly over-predicts the transmission coefficient for small values of $k h$.

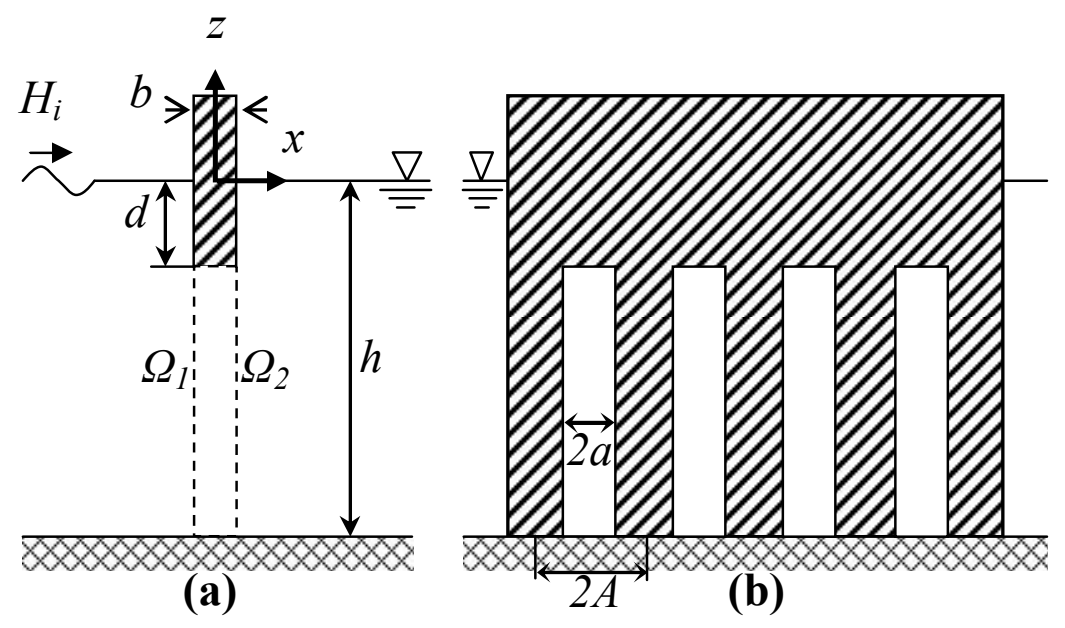

Figure 7. Definition sketch of curtain-wall-pile breakwater; (a) side view, (b) front view.
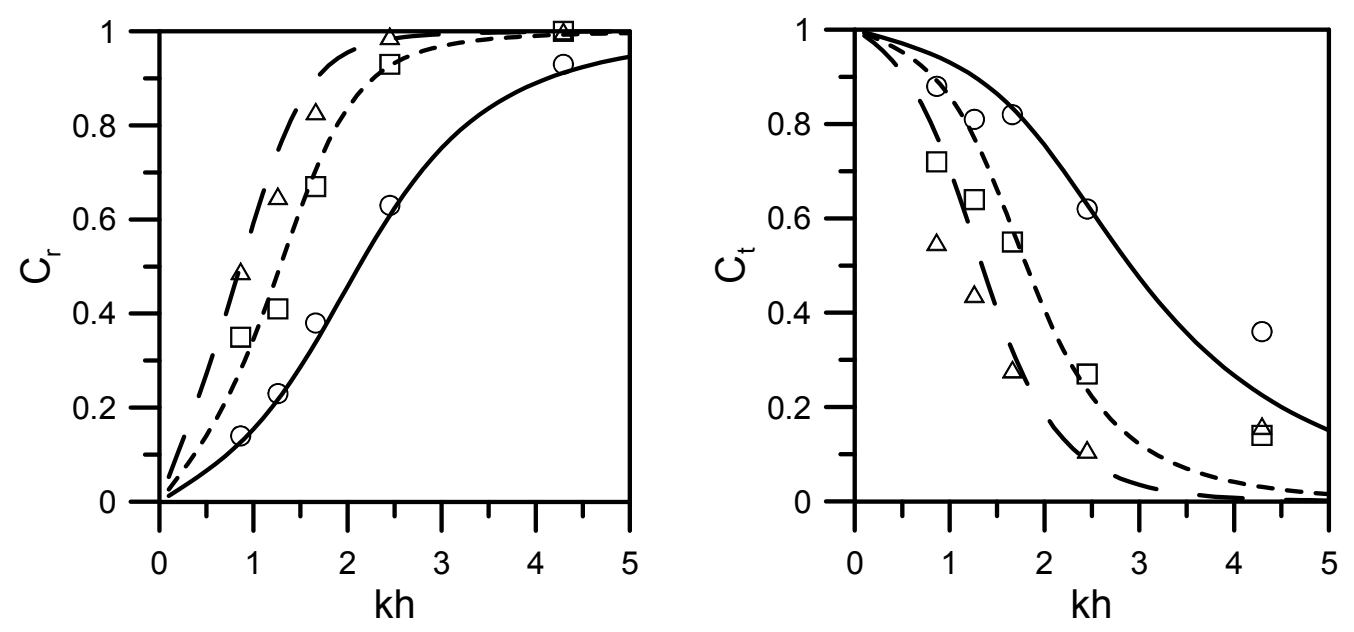

Figure 8. Comparison of predicted reflection and transmission coefficients with experimental results as function of $\mathrm{kh}$ for various drafts of upper wall; $f=0.7$ was used. Predicted: (-) $d / h=0.2$; (----) $d / h=0.4 ;(-\longrightarrow) d / h=0.6$. Measured: (०) $d / h=0.2 ;$ () $d / h=0.4 ;(\triangle) d / h=0.6$. 
The proposed empirical formula is expressed in terms of the structural parameters of the perforated wall and the water depth. It does not need any information for waves. Therefore, it can be used for irregular waves as well. The regular wave model using the eigenfunction expansion method can be extended to irregular waves. Using the regular wave model, the reflection and transmission coefficients can be calculated differently for each frequency component-that is, $C_{r}(f)$ and $C_{t}(f)$ where $f$ is the wave frequency, not the friction coefficient. The spectral densities of the reflected and transmitted waves, respectively, are calculated for a particular frequency component by

$$
\begin{aligned}
& S_{r}(f)=\left|C_{r}(f)\right|^{2} S_{i}(f) \\
& S_{t}(f)=\left|C_{t}(f)\right|^{2} S_{i}(f)
\end{aligned}
$$

where $S_{i}(f)$ is the incident wave energy density. The frequency-averaged reflection and transmission coefficients are then calculated as

$$
\begin{aligned}
& \overline{C_{r}}=\sqrt{\frac{m_{0, r}}{m_{0, i}}} \\
& \overline{C_{t}}=\sqrt{\frac{m_{0, t}}{m_{0, i}}}
\end{aligned}
$$

where $m_{0, i}, m_{0, r}$, and $m_{0, t}$ are the zero-th moments of the incident, reflected, and transmitted wave spectra, respectively.

Suh et al. (2006) also conducted experiments for irregular waves. Fig. 9 compares the measured and predicted wave spectra for the broad, ordinary, and narrow wave spectra in the experiment of Suh et al. (2006), having the peak enhancement factor, $\gamma$, of $1,3.3$, and 10 , respectively. In the figure, the measured incident wave spectra, normalized by the peak energy density, are shown in the uppermost panels. The remaining panels give the measured (solid line) and predicted (dashed line) spectra for reflected and transmitted waves. The reflected and transmitted wave spectra are also normalized by the peak energy density of the incident spectrum. All spectra are plotted as a function of the normalized frequency, $f / f_{p}$, where $f_{p}=1 / T_{p}$ is the peak frequency of the incident wave spectrum. In the figure, also given are the incident significant wave height, $H_{s}$, peak period, $T_{p}$, and the measured and predicted values of frequency-averaged reflection and transmission coefficients. All three of these tests used $d=96 \mathrm{~cm}$.

In all the cases shown, it is seen that the predicted spectra are in generally good agreement with the measured spectra. As with the frequency-averaged reflection and transmission coefficients, the mathematical model slightly over-predicts both wave reflection and transmission, indicating that it slightly underestimates the energy dissipation at the perforated wall.

The above comparisons for regular and irregular waves partially demonstrate that the empirical formula, Eq. (3), could be used to calculate the friction coefficient in the permeability parameter of various types of structures including a perforated wall with vertical slits. 


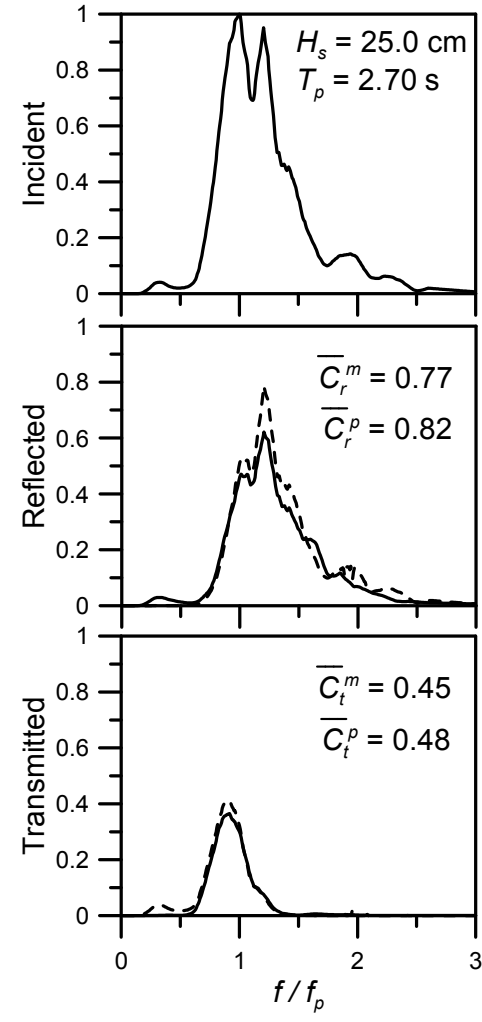

(a)

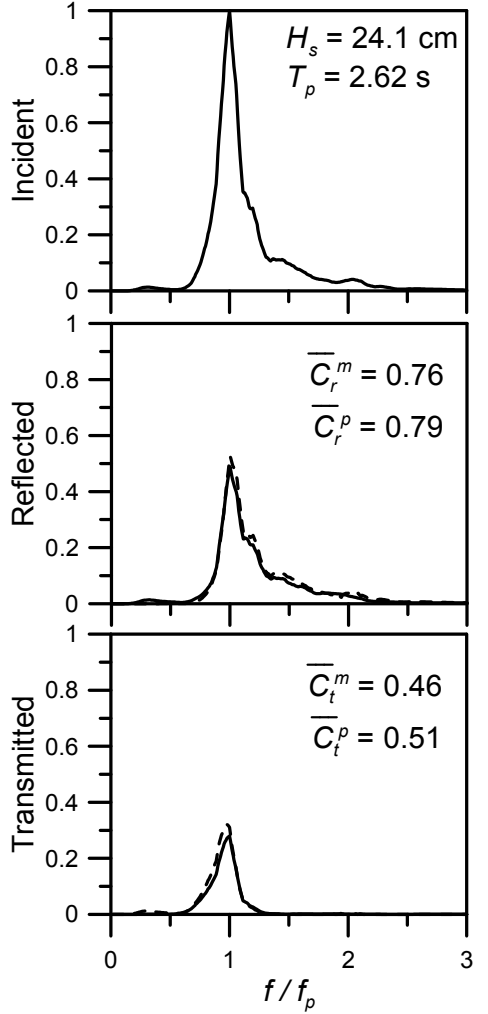

(b)

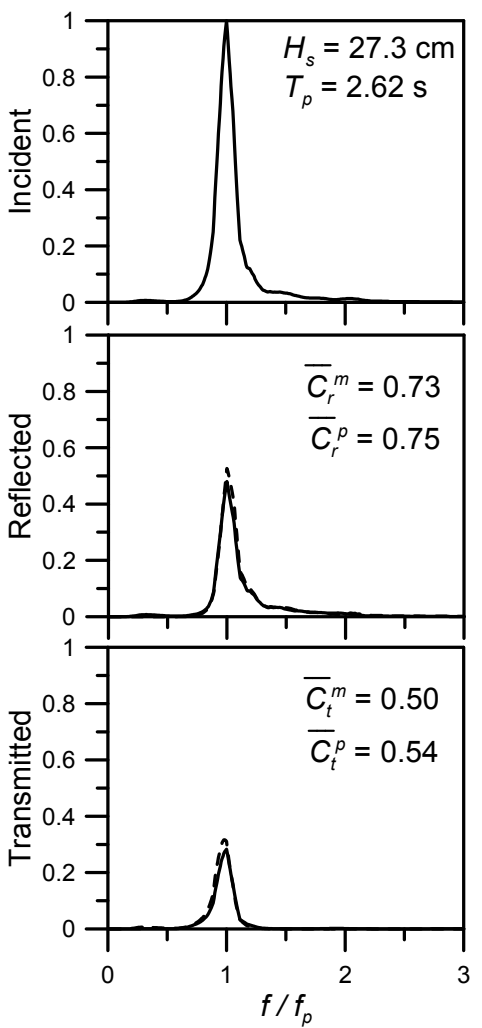

(c)

Figure 9. Comparison between measured (- - ) and predicted (----) spectra of incident, reflected, and transmitted waves: (a) broad spectrum with $\gamma=1.0$; (b) ordinary spectrum with $\gamma=3.3$; and (c) narrow spectrum with $\gamma=10.0$.

\section{CONCLUSION}

In the present study, we proposed an empirical formula for the friction coefficient in the permeability parameter of a perforated wall with vertical slits by using the experimental data of the present study and other researchers. Therefore the friction coefficient $f$ and the permeability parameter $G$ could be directly estimated by Eqs. (2) and (3) with $s=1$ instead of estimating $f$ on the basis of a best fit between measured and predicted values of various hydrodynamic coefficients. The proposed formula was then used to predict the wave reflection and transmission by a pile breakwater or a curtain-wall-pile breakwater. The agreement between the experimental data and calculated results was satisfactory, verifying the appropriateness of the proposed formula. It was also shown that the proposed formula can be used for irregular waves as well.

We showed that the porosity of a perforated wall is also an important variable in the calculation of the friction factor so that the friction coefficient is better expressed as a function of $r b / h$ rather than $b / h$. The porosity of perforated walls constructed in real breakwaters varies in a relatively narrow range, say between 0.2 and 0.4 , indicating that the effect of including porosity in the calculation of the friction coefficient may not be very significant. Multiple perforated plates with gradually decreasing porosity from the frontmost plate are often used to effectively dissipate wave energy at the downwave side of a wave flume. In such a case, the inclusion of porosity may significantly influence the results.

Park et al. (1993) have carried out a laboratory experiment for wave reflection from a perforated wall caisson breakwater. They used different types of perforated walls of the same porosity to find that the difference of reflection coefficients of different types of perforated walls was very small. The empirical formula proposed in this study was developed using the data for perforated walls with vertical slits, but it is expressed in terms of the porosity and thickness of the wall and the water depth. Therefore, the proposed formula could be used for other types of perforated walls with circular holes or 
horizontal slits unless the waves are significantly obliquely incident. Recent researches show that horizontal slits give somewhat less reflection than vertical slits when waves are obliquely incident to a perforated wall because the former gives a larger effective porosity.

\section{ACKNOWLEDGMENTS}

This research was supported by the Korea Sea Grant Program and the Brain Korea 21 Project.

\section{REFERENCES}

Cho, I.-H., and N.-H. Kim. 2002. On an analysis of reflection and transmission coefficients by a vertical slit plate, Journal of Ocean Engineering and Technology, 16, 1-7 (in Korean).

Hagiwara, K. 1984. Analysis of upright structure for wave dissipation using integral equation, Proceedings of $19^{\text {th }}$ International Conference on Coastal Engineering, ASCE, 2810-2826.

Hossain, A., W. Kioka, and T. Kitano. 2001. Transmission of long waves induced by short-wave groups through a composite breakwater, Coastal Engineering Journal, 43, 83-97.

Huang, Z. 2007a. An experimental study of wave scattering by a vertical slotted barrier in the presence of a current, Ocean Engineering, 34, 717-723.

Huang, Z. 2007b. Wave interaction with one or two rows of closely spaced rectangular cylinders, Ocean Engineering, 34, 1584-1591.

Isaacson, M., S. Premasiri, and G. Yang. 1998. Wave interactions with vertical slotted barrier, Journal of Waterway, Port, Coastal, and Ocean Engineering, ASCE, 124, 118-126.

Kakuno, S., and P.L.-F. Liu. 1993. Scattering of water waves by vertical cylinders, Journal of Waterway, Port, Coastal, and Ocean Engineering, ASCE, 119, 302-322.

Kim, B.H. 1998. Interactions of Waves, Seabed and Structures, PhD dissertation, Seoul National Univ., Seoul, Korea.

Kriebel, D.L. 1992. Vertical wave barriers: wave transmission and wave force, Proceedings of $23^{\text {rd }}$ International Conference on Coastal Engineering, ASCE, 1313-1326.

Li, Y., Y. Liu, and B. Teng. 2006. Porous effect parameter of thin permeable plates, Coastal Engineering Journal, 48, 309-336.

Losada, I.J., M.A. Losada, and A. Baquerize. 1993. An analytical method to evaluate the efficiency of porous screens as wave dampers, Applied Ocean Res., 15, 207-215.

Mei, C.C., P.L.-F. Liu, and A.T. Ippen. 1974. Quadratic loss and scattering of long waves, Journal of Waterways, Harbors Coastal Eng. Div., ASCE, 100, 217-239.

Park, W.S., I.S. Chun, and D.S. Lee. 1993. Hydraulic experiments for the reflection characteristics of perforated breakwaters, Journal of Korean Society of Coastal and Ocean Engineers, 5, 198-203 (in Korean, with English abstract).

Sollitt, C.K., and R.H. Cross. 1972. Wave transmission through permeable breakwaters, Proceedings of $13^{\text {th }}$ International Conference on Coastal Engineering, ASCE, 1827-1846.

Suh, K.D., W.S. Park, and B.S. Park. 2001. Separation of incident and reflected waves in wave-current flumes, Coastal Engineering, 43, 149-159.

Suh, K.-D., S. Shin, and D.T. Cox. 2006. Hydrodynamic characteristics of pile-supported vertical wall breakwaters, Journal of Waterway, Port, Coastal, and Ocean Engineering, ASCE, 132, 83-96.

Willmott, C.J. 1981. On the validation of models, Physical Geography, 2, 184-194.

Yu, X. 1995. Diffraction of water waves by porous breakwaters, Journal of Waterway, Port, Coastal, and Ocean Engineering, ASCE, 121, 275-282.

Zhu, S., and A.T. Chwang. 2001. Analytical study of porous wave absorber, Journal of Engineering Mechanics, ASCE, 127, 326-332. 